\title{
Immortalized Mouse Inner Ear Cell Lines Demonstrate a Role for Chemokines in Promoting the Growth of Developing Statoacoustic Ganglion Neurons
}

\author{
Lynne M. Bianchi, ${ }^{1,2}$ Zeeba Daruwalla, ${ }^{1}$ Therese M. Roth, ${ }^{2}$ Naweah P. Attia, ${ }^{2}$ \\ Nicholas W. Lukacs, ${ }_{2,4,5}^{3}$ Ayo-Lynn Richards, ${ }^{1,2}$ Ian O. White, ${ }^{1}$ Susan J. Allen, ${ }^{2}$ \\ AND KATE F. BARALD $2,4,5$ \\ ${ }^{1}$ Neuroscience Department, Oberlin College, Oberlin, OH, USA \\ ${ }^{2}$ Department of Cell and Developmental Biology, University of Michigan Medical School, Ann Arbor, MI, USA \\ ${ }^{3}$ Department of Pathology, University of Michigan Medical School, Ann Arbor, MI, USA \\ ${ }^{4}$ Program in Neuroscience, University of Michigan Medical School, Ann Arbor, MI, USA \\ ${ }^{5}$ Cell and Molecular Biology, University of Michigan Medical School, Ann Arbor, MI, USA
}

Received: 30 November 2004; Accepted: 20 July 2005; Online publication: 21 October 2005

\begin{abstract}
The target-derived factors necessary for promoting initial outgrowth from the statoacoustic ganglion (SAG) to the inner ear have not been fully characterized. In the present study, conditioned medium from embryonic Immortomouse inner ear cell lines that maintain many characteristics of developing inner ear sensory epithelia were screened for neurite-promoting activity. Conditioned medium found to be positive for promoting SAG neurite outgrowth and neuronal survival was then tested for the presence of chemokines, molecules that have not previously been investigated for promoting SAG outgrowth. One candidate molecule, monocyte chemotactic protein 1 (MCP-1), was detected in the conditioned medium and subsequently localized to mouse hair cells by immunocytochemistry. In vitro studies demonstrated that function-blocking MCP-1 antibodies decreased the amount of SAG neurite outgrowth induced by the conditioned medium and that subsequent addition of MCP-1 protein was able to promote outgrowth when added to the antibodytreated conditioned medium. The use of the Immortomouse cell lines proved valuable in identifying this
\end{abstract}

Correspondence to: Lynne M. Bianchi • Neuroscience Department • Oberlin College, Science Center A243, Oberlin, OH 44074, USA. Telephone: +1-440-7758369; email: lynne.bianchi@oberlin.edu candidate cofactor that promotes outgrowth of earlystage SAG nerve fibers and is expressed in embryonic hair cells.

Keywords: auditory, cochlea, neural, development, spiral ganglion, Immortomouse

\section{INTRODUCTION}

Inner-ear-derived growth factors. Target-derived growth and survival factors are critically important for the development of neuronal populations. Such factors also appear to regulate innervation of the developing inner ear at the otocyst stage (reviewed by Barald and Kelley 2004). During early stages of embryogenesis [embryonic day (E) 4-6 chick; E11-14 mouse, rat], the otocyst releases (a) soluble factor(s) that promote the outgrowth and survival of innervating statoacoustic ganglion (SAG) neurons (Ard and Morest 1984; Ard et al. 1985; Lefebvre et al. 1990; Bianchi and Cohan 1991, 1993a). The otocyst-derived factors (ODF) from chick, mouse, and rat have similar bioactivity, and each can promote outgrowth from the other species. For example, cultured E1214 mouse inner ears secrete ODF that promotes outgrowth of E4-6 chick SAG, and cultured E4-6 chick inner ears secrete ODF that promotes outgrowth of E12-14 mouse SAG (Bianchi and Cohan 1993a). Thus, ODF refers to the factor(s) released 
from the early-stage otocyst of any of these species. The identities of the biologically active components of ODF, which appear to be critical during early stages of neurite outgrowth, have not been fully characterized.

In contrast to the initial stages of neurite outgrowth, as the SAG ultimately separates to form the distinct cochlear and vestibular ganglia, each of these neural populations begins to rely on specific, identified growth factors. Beginning at the time of synaptogenesis (approximately E13.5, in mouse), the majority of cochlear neurons becomes dependent on neurotrophin-3 for survival, whereas vestibular neurons rely primarily on brain-derived neurotrophic factor (BDNF) (reviewed by Fritzsch et al. 1997). However, prior to this period, when afferent fibers are first extending toward the target cells in the periphery, these neurotrophins alone are not sufficient for the survival or outgrowth of SAG neurons (Bianchi and Cohan 1993a; Bianchi et al. 1998; Tessarollo et al. 2004). Other studies have also suggested that FGFs and BDNF play a sequential role in regulating aspects of SAG migration, differentiation, and outgrowth (Hossain et al. 1997; Brumwell et al. 2000).

Previous studies compared ODF to other growth factors in an attempt to identify the active components of ODF. Comparisons with a large number of growth factors including the neurotrophins, ciliary neurotrophic factor (CNTF), fibroblast growth factors (FGFs), transforming growth factors (TGFs) and tumor necrosis factors (TNFs) failed to identify a candidate molecule for ODF (Bianchi and Cohan 1993a,b). Although these assays did not indicate that FGFs alone mimicked ODF, other laboratories have reported a role for FGFs in regulating aspects of SAG neuroblast migration and neurite outgrowth (Hossain and Morest 2000). In our assays, only CNTF promoted neurite outgrowth of SAG explants similar to that observed with chick ODF-conditioned medium. However, CNTF had no effect on survival or outgrowth of dissociated SAG neurons, suggesting that its effect on explants was indirect, perhaps through interactions with the nonneuronal cells present in the ganglion explant (Bianchi and Cohan 1993a). Furthermore, ODF did not demonstrate any CNTF-like bioactivity (Bianchi and Cohan 1993a; Bianchi et al. 1998).

A cytokine or chemokine other than CNTF may normally influence SAG outgrowth in vivo. Chemokine proteins were initially identified for their ability to attract and activate immune cells by interacting with specific receptors (Coughlan et al. 2000). Recent studies have demonstrated that chemokines are also present in developing neurons and have suggested that these molecules may also play roles in neuronal migration, outgrowth, or survival (Meng et al. 1999; Chalasani et al. 2003a,b; Belmandi et al. 2005). In some cases, the chemokines were found to regulate axonal outgrowth by inhibiting the repellent effects of molecules such as semaphorins and slit-2 (Chalasani et al. 2003b). This is the first report documenting the expression and function of chemokines in the developing inner ear.

Sources of SAG neurite-promoting factors. Due to the small size of the otocyst it is difficult to generate sufficient quantities of otocyst-conditioned medium for biochemical analysis from the organ itself. Thus, another tissue source is needed. Earlier studies found that conditioned medium from tissues such as limb bud and heart did not contain SAG neurite-promoting factor(s) (Bianchi and Cohan 1991); therefore a means of obtaining larger quantities of conditioned medium from inner ear cells would aid biochemical characterization and purification efforts.

The present study tested whether conditioned medium from conditionally immortalized otocyst cell lines derived from the transgenic Immortomouse (Barald et al. 1997; Bianchi and Barald 1998; Thompson et al. 2003; Germiller et al. 2004) provides a source of ODF-like activity. The cells used in the present study were derived from embryonic day (E) 9.5 otocysts as previously described (Barald et al. 1997; Germiller et al. 2004). A total of 307 IMO cell lines were cloned from these otocysts and several have previously been characterized (Thompson et al. 2003; Germiller et al. 2004). Eight IMO cell lines were identified as positive for hair cell markers such as myosin VIIa, as well as supporting cell marker Hes 1 (Germiller et al. 2004). However, individual cells in these cloned lines were found to express $\mathrm{HC}$ and SC markers simultaneously in culture. These cell lines, which are derived from the very early otocyst, appear to represent pluripotent precursor cell lines that are common precursors to $\mathrm{HC}$ and SC, and possibly SAG neurons (Germiller et al. 2004).

In the present study, conditioned medium was screened from five IMO cell lines that express hair cell and supporting cell markers (Germiller et al. 2004). Four of the five lines tested were able to promote neurite outgrowth from SAG explants. The fifth cell line was negative for bioactivity and served as a negative control in subsequent experiments. The survival and neurite-promoting properties of one cell line, IMO2B1, were then compared to the known characteristics of ODF. IMO2B1-conditioned medium was also screened for the presence of cytokines and chemokines. Monocyte chemoattractant protein1 (MCP-1; originally named JE in mouse) was secreted by IMO2B1 cells and importantly was subsequently detected in mouse embryonic hair cells. Antibodies to MCP-1 significantly decreased IMO2B1-conditioned medium-induced SAG neurite outgrowth as well as the chemoattraction of monocytes exposed to 
TABLE 1

Scoring criteria for SAG explants

\begin{tabular}{|c|c|c|c|c|c|c|}
\hline Score & 0 & 1 & 2 & 3 & 4 & 5 \\
\hline Criteria & $\begin{array}{l}\text { No growth, } \\
\text { may be } \\
\text { small patch } \\
\text { NNC, may } \\
\text { be retracting } \\
\text { fibers present }\end{array}$ & $\begin{array}{l}\text { Short, sparse } \\
\text { growth, mix fibers } \\
\text { and NNC, up to } \\
1 / 4-3 / 4 \text { around } \\
\text { explant }\end{array}$ & $\begin{array}{l}\text { Approx. } 1 / 4 \text { explant } \\
\text { extending medium- } \\
\text { long growth, mix } \\
\text { of fibers and NNC }\end{array}$ & $\begin{array}{l}\text { Approx. } 1 / 2 \\
\text { explant } \\
\text { surrounded by } \\
\text { medium-long, } \\
\text { dense, radial } \\
\text { growth, mix of } \\
\text { fibers and NNC }\end{array}$ & $\begin{array}{l}\text { Approx. } 3 / 4 \text { dense, } \\
\text { radial growth, mix } \\
\text { of fibers and NNC, } \\
\text { fibers at least } 1 \\
\text { explant diameter } \\
\text { in length }\end{array}$ & $\begin{array}{l}\text { Long, dense } \\
\text { radial growth } \\
\text { surrounding } \\
\text { explant, all } \\
\text { fibers at least } \\
1 \text { explant } \\
\text { diameter in } \\
\text { length }\end{array}$ \\
\hline
\end{tabular}

NNC, Nonneuronal cells.
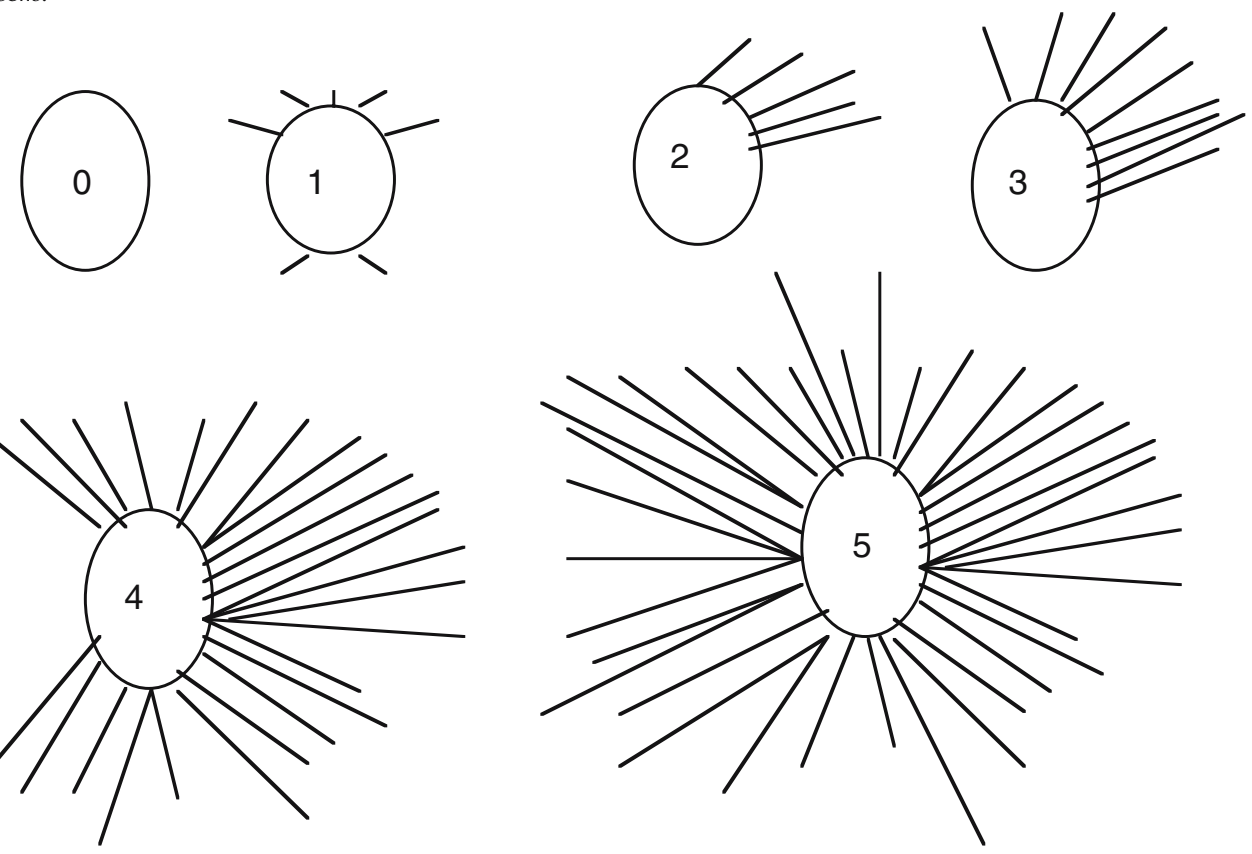

the IMO2B1-conditioned medium. The results of the present study demonstrate that IMO2B1 produces components that share many characteristics of ODF and that MCP-1 is a candidate SAG outgrowthpromoting cofactor.

\section{METHODS}

\section{Cell cultures}

Otocyst cell lines. Otocyst cell lines were generated from the H2kbtsA58 transgenic mouse (Immortomouse) as previously described (Barald et al. 1997; Thompson et al. 2003; Germiller et al. 2004). Cells from specific IMO lines were initially grown at $32^{\circ} \mathrm{C}$ in chick embryo fibroblast (CEF) medium on Primaria culture plates $(100 \mathrm{~mm}$, Falcon) to allow IMO cells to proliferate. The proliferating cells were used to produce a conditioned growth medium, some of which was then diluted 1:1 (v/v) with CEF-fetal bovine serum (FBS) $-\gamma$-interferon-containing medium. Other cells were grown in CEF without serum

for 4-21 days before harvesting the conditioned medium. Gamma interferon, however, was replenished every 4 days. Both serum-free and serum-containing formulations produced conditioned medium that promoted the survival and outgrowth of SAG neurons. Control medium containing only serum $(1-10 \%)$ did not promote any outgrowth of SAG. For the SAG cultures described in this report and for biochemical characterization, the cell lines were grown under serum-free conditions. Conditioned media were harvested and stored at $4^{\circ} \mathrm{C}$ (up to 4 months) until use. Multiple batches of conditioned medium from different isolates of the same cell lines were used for the assays in the current study. Individual batch numbers are indicated for each experiment.

Neuronal cultures. Explants of SAG (E5-6 chick or E14 mouse) were prepared as described previously (Bianchi and Cohan 1993a). Briefly, explants were grown on poly-D-lysine-coated wells (48-well plates, CoStar) for 24-36 $\mathrm{h}$ then scored for growth. SAG explants were scored on a standard scale of $0-5$ 
(Table 1). Criteria for each score were used uniformly for each ganglion examined and scored by two or more investigators. The majority of experiments were done with chick SAG because they are the most easily obtained for screening bioactivity. Previous studies had found that chick SAG respond equally well to both chick and mouse ODF and that chick and mouse ODF promote outgrowth similarly (Bianchi and Cohan 1993a). To screen the activity of IMOconditioned medium, $200 \mu \mathrm{l}$ of conditioned medium was added to each ganglion. Controls were treated with either defined medium (below) or the medium used to culture IMO cells (lacking gammainterferon, above). Both methods produced similar results. Human and mouse recombinant MCP-1 (R\&D Systems, Minneapolis, MN) was added at a concentration of $10-1000 \mathrm{ng} / \mathrm{ml}$ diluted in defined medium. Function-blocking anti-mouse MCP-1 antibody (AF-479-NA, R\&D Systems) was added to IMO2B1conditioned medium at concentrations of $10-300$ $\mathrm{ng} / \mathrm{ml}$. Control IgG (goat, R\&D Systems) was added to the conditioned medium at concentrations of $1-200 \mu \mathrm{g} / \mathrm{ml}$.

Explants of E8 chick dorsal root ganglia (DRG) were plated on polylysine-coated wells as described previously (Bianchi and Cohan 1993a) and treated with human recombinant BDNF (10 ng/ml, Regeneron Pharmaceuticals). Mean radial outgrowth was scored as for SAG explants (Table 1).

Dissociated SAG cells were prepared by pooling 20-30 ganglia and incubating them in $0.1 \%$ trypsin (Sigma) in $\mathrm{Ca}^{2+} / \mathrm{Mg}$-free phosphate-buffered saline (PBS) for $10 \mathrm{~min}$ at $37^{\circ} \mathrm{C}$. Ganglia were then rinsed in $\mathrm{Ca}^{2+} / \mathrm{Mg}$-free PBS and incubated in $0.1 \%$ trypsin inhibitor (Sigma) for $10 \mathrm{~min}$ at $37^{\circ} \mathrm{C}$. Ganglia were rinsed again, then resuspended in defined culture medium [Dulbecco's modified Eagle's medium (DMEM)/F-12 supplemented with ITS+ (Collaborative Research)]. Cells were dissociated with a firepolished pipet and plated on poly-D-lysine-coated plates at a density of 30,000 cells per well (48-well plates, CoStar).

To estimate cell survival, cells were fixed in $10 \%$ neutral buffered formalin for $10 \mathrm{~min}$ then immunolabeled with an anti-neurofilament antibody (NF 150, Chemicon). Following fixation, cells were treated with hydrogen peroxide, rinsed in PBS, then blocked with $1 \%$ normal goat serum. Cells were incubated overnight in primary, anti-neurofilament antibody $(1: 1000)$ at $4^{\circ} \mathrm{C}$. The following day, cells were rinsed carefully with PBS to prevent dislodging adherent cells. Cells were then incubated in biotinylated secondary antibody followed by the avidin-biotin complex (ABC, Vector Laboratories). Sites of binding were visualized with diaminobenzidine (DAB, Sig$\mathrm{ma})$. The percentage of neurofilament-positive cells was calculated from ten randomly selected fields throughout the well.

\section{Protein analysis}

Protein array analysis. The presence of cytokines was initially screened using TransSignal RayBio Mouse Cytokine Antibody Array Kits (Panomics). Two arrays are provided in each kit. For each of the three protein array experiments conducted in the current study, one membrane was used for control media (unconditioned media) and the other for IMO2B1 media that was found to promote SAG outgrowth (batches 6.20, 9.13, 9.22). In one experiment, the control membrane was sectioned so that control media and IMO3A1 conditioned media (negative control conditioned medium) could be run simultaneously with the IMO2B1-conditioned media. To assay for chemokines, IMO-conditioned medium or control medium was incubated on the array membranes for $1 \mathrm{~h}$. The membranes were washed and the biotinylated anti-cytokine antibody mixture was added for $2 \mathrm{~h}$ at room temperature. The membranes were then washed and incubated in streptavidin-HRP conjugate for $1 \mathrm{~h}$ at room temperature followed by detection with chemiluminescent substrate (Pierce).

Western blot analysis. IMO2B1 supernatants were concentrated by one of two methods. Conditioned medium (1-5 ml) was concentrated over either a 5or 10-kDa filter (Amicon or Centricon, respectively) for $2-2.5 \mathrm{~h}$, or $50 \mathrm{ml}$ was concentrated over a protein spin concentrator (Amicon; 10-kDa cutoff) for $30 \mathrm{~min}$. These concentrated samples and positive controls, recombinant human MCP-1 and recombinant mouse MCP-1 (R\&D Systems), were run on 10$20 \%$ Tris tricine gels (BioRad) for $70 \mathrm{~min}$ at $100 \mathrm{~V}$ followed by blot transfer onto a polyvinylidene difluoride membrane (BioRad) for $1 \mathrm{~h}$ at $100 \mathrm{~V}$. The membrane was blocked in bovine serum albumin, then rinsed in Tris-buffered saline (TBS) with $0.05 \%$ Tween (TTBS) and incubated in primary antibody (anti-mouse MCP-1/JE, R\&D Systems, AE $479 \mathrm{NA})$ overnight at $4^{\circ} \mathrm{C}$. The membranes were rinsed in TTBS and then incubated in secondary antibody (peroxidase-conjugated rabbit anti-goat antibody, Jackson ImmunoResearch, 1:10,000) at $4^{\circ} \mathrm{C}$ overnight. The membranes were then rinsed in TTBS and TBS then exposed to the Westpico chemiluminescent substrate (Pierce) and subsequently exposed to film (Pierce). Control blots were processed simultaneously as above, excluding the primary antibody.

Immunocytochemistry. Cultured E14 mouse inner ear cells were double-labeled for MCP-1 and the 
hair cell marker myosin VI. Dissociated embryonic day 14 (E14) mouse inner ears were cultured as described previously (Bianchi et al. 2002). In this preparation inner ear cells reaggregate into distinct patterns within $24 \mathrm{~h}$ in vitro. One aggregate type, the dome, is composed of both hair cells and supporting cells as identified with hair cell and supporting cell markers (Bianchi et al. 2002). Hair cells are generally located more centrally, with supporting cells surrounding the hair cells. Briefly, inner ear cells were fixed in formalin, rinsed in PBS, blocked in normal serum and incubated overnight in primary antibodies (anti-myosin VI, 1:50, T. Hasson, UCSD; anti-MCP-1, 1:10, anti-TNF alpha 1:10, anti-RANTES 1:10; R\&D Systems,). The following day, cultures were rinsed, incubated in fluorescent secondary antibodies (Texas Red, fluorescein, 1:50, Jackson ImmunoResearch) for $2 \mathrm{~h}$ then viewed with a Zeiss LSM-5 confocal microscope.

Monocyte chemotactic assay. To confirm the chemotactic activity of the MCP-1 protein and the functionblocking abilities of the anti-MCP-1 antibody used in the SAG assays, a classic monocyte chemotactic assay was used. The mononuclear cells were isolated from whole blood $(50 \mathrm{ml})$ drawn from one of the investigators (NWL) under protocols approved by the University of Michigan Institutional Review Board. Whole blood was mixed 1:1 with normal saline (Merck). Ficoll-Hypaque (FH, 15 ml, Beckman-Coulter) was layered into each of six 50-ml conical polypropylene tubes (Corning) and the blood/saline mixture $(15 \mathrm{ml})$ was carefully layered over the $\mathrm{FH}$. Tubes were centrifuged in a Beckman refrigerated centrifuge at $400 \times g$ for $30 \mathrm{~min}$ at $20^{\circ} \mathrm{C}$. The plasma layer was removed by careful pipetting. The mononuclear layer (buffy coat) was placed in separate tubes $(25 \mathrm{ml})$ and the total volume in each tube was brought to $50 \mathrm{ml}$ with $\mathrm{PBS}$. The tubes were centrifuged at $900 \times g$ for $10 \mathrm{~min}$ at $4^{\circ} \mathrm{C}$, the supernatant was discarded and the pellet resuspended in $50 \mathrm{ml}$ of F-12 medium (Invitrogen). Centrifugation and washes were repeated three times. The final pellet was resuspended in $5 \mathrm{ml}$ of F-12 medium and $50 \mu \mathrm{l}$ was used for trypan blue exclusion and cell counting. The remaining cells were resuspended in a total of $6.66 \mathrm{ml}$ of F-12 to bring the total to $3 \times 10^{6}$ viable cells $/ \mathrm{ml}$.

The monocyte samples to be tested for chemotaxis were pipetted at a volume of $162 \mu \mathrm{l}$ per well in at least triplicate wells in a 12-well chemotaxis chamber (Ann Arbor Plastics Co.) An 8- $\mu \mathrm{m}$ pore-size polycarbonate filter (polyvinylpyrrolidone-free, Nucleopore Corp.) was placed over the wells, and the flexible gasket secured with the top chamber by screws. The monocyte-containing blood cell suspension $(100 \mu \mathrm{l})$ was placed into the top wells and the chambers placed in a $37^{\circ} \mathrm{C}$ humidified $\mathrm{CO}_{2}$ incubator for $1 \mathrm{~h}$. The top chamber and remaining cells were then were removed, the upper side of the membrane scraped with a cell scraper to remove nonmigratory cells, and the membrane was hung to dry at room temperature for $1 \mathrm{~h}$. At the end of that time, the membranes were fixed in $\mathrm{MeOH}$ for $10 \mathrm{~min}$, stained with $2 \%$ toluidine blue, and then counterstained with hematoxylin and eosin. Counts of the monocytes among the cells that had migrated to the underside of the membrane were taken from ten random fields using an Olympus microscope in bright field at $1000 \times$ magnification . Chemotactic activity was expressed as the mean number of migrated monocytes per total of ten fields in $n=3-7$ replicates of each group tested for cytokine activity, less the background number of cells that migrated through the filter in medium-only controls. Background was calculated from the two control media (F-12, the base medium in CEF or in Optipro, the serum-free medium; Invitrogen). The reagents tested in this experiment were used at the concentrations recommended by the manufacturer (R\&D Systems). The assay included recombinant human MCP-1/CCL2 (20 ng/ml, R\&D Systems, 279MC, lot no. NS023021); MCP-1 preneutralized with anti-mouse MCP-1/JE antibody (5 $\mu \mathrm{g} / \mathrm{ml}, \mathrm{R} \& \mathrm{D}$ Systems, AF-479-NA, lot no. 192403). IMO2B1-conditioned medium that had previously been shown to be positive in the SAG outgrowth assay was also tested in the chemotaxis assays (both with and without the addition of anti-MCP-1 antibody). Control wells of undiluted F-12 (Invitrogen, $n=4$ ) medium alone or Optipro medium (Invitrogen, $n=3$ ) were used to establish a background level of monocyte chemotaxis. The average number of monocytes in control conditions (58, $n=7$ ) was subtracted from each experimental group prior to graphing the data.

Statistical analysis. Student's $t$ tests were used to make comparisons between groups. $P$ values of less than 0.05 were used as the level of statistical significance.

\section{RESULTS}

Inner ear cell lines secrete neurite outgrowth-promoting factors

To screen for bioactive factors in conditioned medium from IMO cell lines, SAG explants from E5 chick embryos were plated on poly-D-lysinecoated dishes. The explants were then treated with $200 \mu \mathrm{l}$ of serum-free IMO-conditioned medium or control medium that was not exposed to IMO cells. After $24 \mathrm{~h}$ in culture, the explants were scored using a scale of 1-5 (Table 1). Individual explants treated 

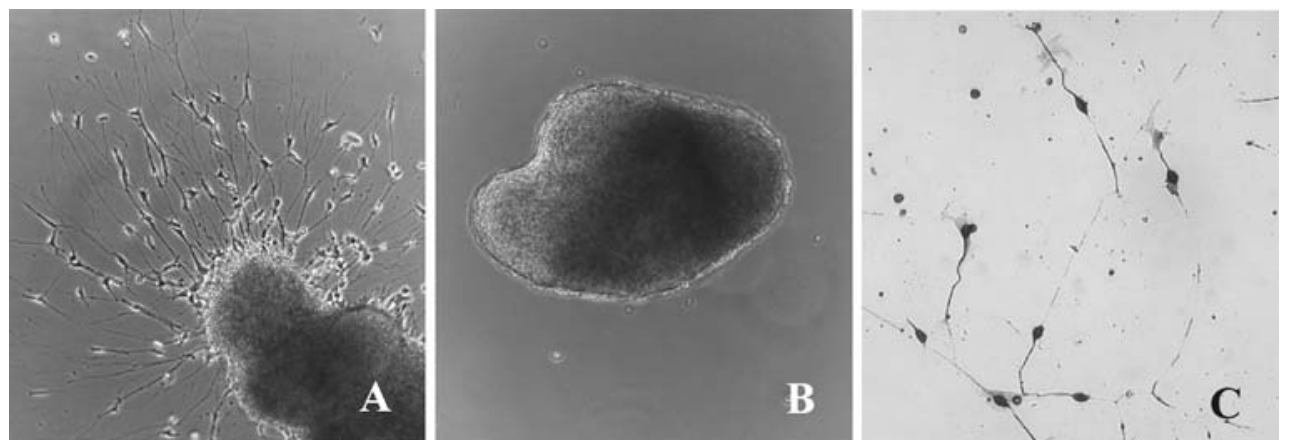

FIG. 1. (A) E5 chick SAG grown in the presence of IMO2B1conditioned medium exhibits extensive neurite outgrowth (explant score $=4$, batch 9.22). (B) E5 chick SAG grown in control (unconditioned) medium does not produce outgrowth (explant score $=0$ ).

with IMO2D2, IMO1G1, IMO3C3, or IMO2B1-conditioned medium produced outgrowth with scores ranging from 2.0 to 5.0; with mean scores of 2.5-3.4 ( $n=23-25$ SAG per condition; Fig. 1A). In contrast, explants grown in IMO3A1 or control (unconditioned) medium produced outgrowth of less than 0.5 ( $n=12$; Fig. 1B). These results demonstrated that conditioned medium from four of the five IMO cell lines tested produced outgrowth significantly greater than control medium $(p<0.01)$. The IMO2B1conditioned medium (batches 6.20, 9.22) consistently produced the most extensive outgrowth (mean score 3.4). The extent and morphology of outgrowth was similar to that previously observed with chickand mouse-derived ODF (Bianchi and Cohan 1993a). Thus, IMO2B1-conditioned medium was selected for subsequent analysis.

IMO2B1 cells produce factors that influence SAG neuron survival. To calculate the neuronal survival obtained with conditioned medium from the IMO2B1 cell line, dissociated E5 chick SAG neurons were assayed. Survival of SAG in the presence of IMO2B1conditioned medium (batch 1.90) was estimated by

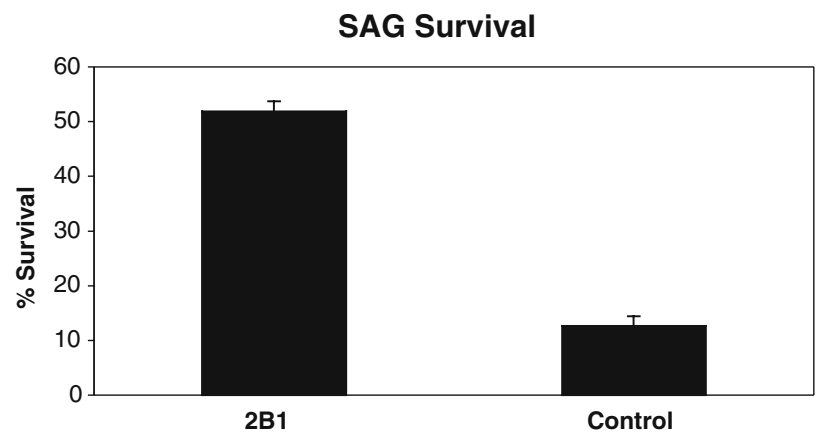

FIG. 2. Survival of dissociated E5 chick SAG neurons in IMO2B1 (batch 1.90) was significantly higher than SAG grown in control medium. $(p<0.01 ; \pm$ SE).
Magnification, $\times 100$. (C) Dissociated E6 chick SAG neurons grown in IMO2B1-conditioned medium (batch 1.90) survive and extend long neurites. Magnification, $\times 100$.

counting the number of neurofilament positive cells after $24 \mathrm{~h}$ in vitro (Fig. 1C). In the presence of this IMO2B1-conditioned medium, $52 \%$ of the SAG neurons were neurofilament positive ( $n=12$ wells). This was significantly greater than control cultures in which only $13 \%$ of the cells were neurofilament positive $(p<0.01, n=6$ wells; Fig. 2$)$. The survival obtained with IMO2B1-conditioned medium was similar to that obtained previously with ODF (Bianchi and Cohan 1993a). Together, the bioassay experiments found ODF-like activity in IMO2B1-conditioned medium in both neurite-promoting and survival assays.
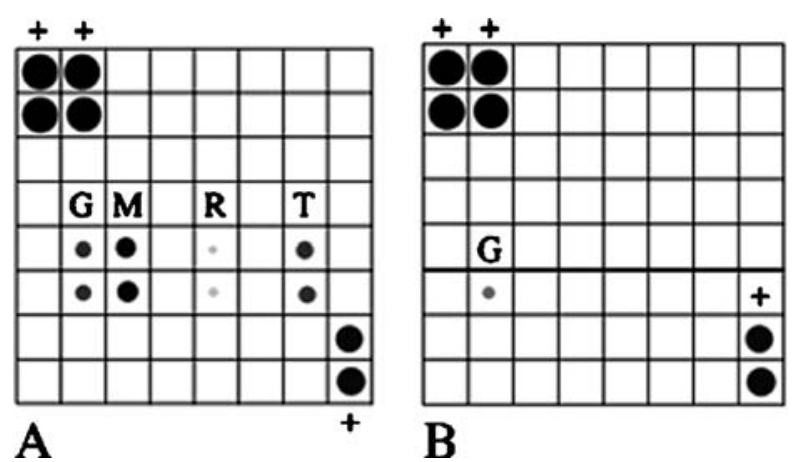

FIG. 3. Protein array analysis of (A) IMO2B1-conditioned media and (B) control medium. An array was section in order to test unconditioned medium and negative control IMO3A1 at the same time (B). Control medium that was not exposed to IMO cells (top, B) and IMO3A1-conditioned medium that failed to promote neurite outgrowth from chick SAG (batch 11.23; bottom, B) only detected the manufacturer's positive controls (biotinylated HRP conjugate, +) and low levels of gamma interferon (G). All other cytokines were below the level of detection in these media. In contrast, soluble TNF receptor $1(\mathrm{~T})$, gamma interferon $(\mathrm{G}), \mathrm{MCP}-1(\mathrm{M})$, and low levels of RANTES were detected in IMO2B1-conditioned medium shown to promote neurite outgrowth (A, batch 9.13). (- Indicates negative controls, + positive controls). 

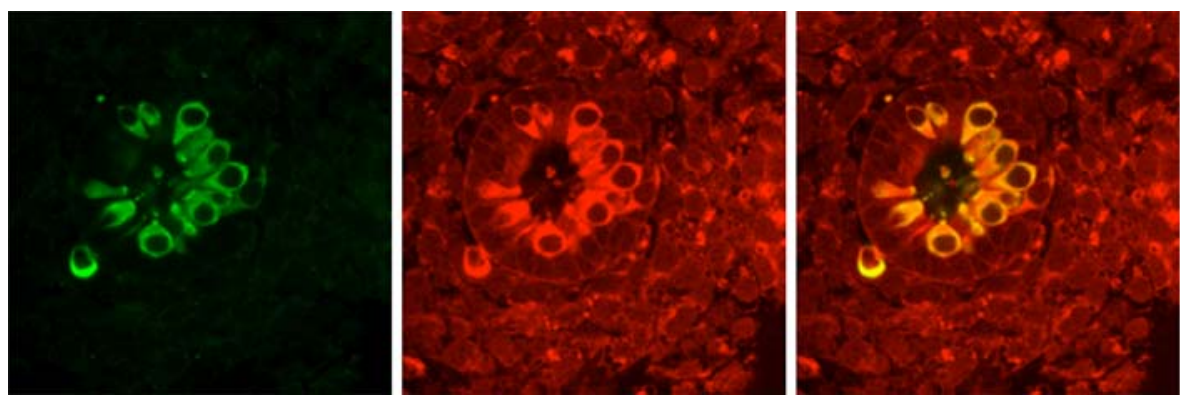

FIG. 4. Immunofluorescence (green) representing the secondary antibody binding to the hair cell marker myosin VI was detected in hair cells of dissociated E14 mouse inner ear cultures. The dissociated cells form aggregates of hair cells and supporting cells. MCP-1 was also prominently expressed in hair cells (red), as well as in some

\section{Chemokines are present in IMO2B1-conditioned medium and inner ear cells}

Chemokines are detected by protein array analysis. IMO2B1-conditioned medium was screened for the presence of cytokines/chemokines using the TranSignal RayBio antibody array (Panomics). IMO3A1, which did not promote SAG outgrowth, was used as a control (batch 11.23; Fig. 3B, bottom). MCP-1, which can be detected as low as $5 \mathrm{pg} / \mathrm{ml}$ on the array, was prominently expressed in three independent trials with different batches of IMO2B1 (batches 6.20, 9.13, 9.22). MCP-1 was consistently the most highly expressed protein present on the arrays incubated with IMO2B1-conditioned medium. Very low levels of RANTES were faintly detected in all three batches of IMO2B1, although this low level of expression was not always apparent in the photomicrographs. RANTES can be detected at a level of $50 \mathrm{pg} / \mathrm{ml}$ on the array. Low levels of TNF alpha were detected in two out of the three trials (batches 6.20,9.22), whereas soluble TNF receptor 1 (TNFR1) was detected in one of the trials (batch 9.13, Fig. 3A). Gamma interferon, which is present in the medium added to IMO cultures, was also detected in all IMO2B1 batches as expected since the cells are grown in this medium to ensure proliferation (Fig. 3A). Gamma interferon also appeared to be more highly expressed than in control medium (below). The $\gamma$-interferon used in this study (Chemicon) is a recombinant mouse interferon expressed in E. coli and subsequently affinity purified by the manufacturer and tested for impurities and the absence of other contaminating cytokines. No such contaminants were present in the $\gamma$-interferon. The other cytokines present on the blot were below the level of detection in IMO2B1-conditioned medium. These included G-CSF, GM-CSF, IL2, IL-3, IL-4, IL-5, IL-6, IL-9, IL-10, IL-12, IL-12p70, IL-13, IL-17, MCP-5, CSF, TPO, and VEGF.

In contrast to IMO2B1-conditioned medium, IMO3A1-conditioned (batch 11.23) medium did not unidentified cells surrounding the aggregate. Supporting cells within the aggregate were negative for MCP-1. The third panel demonstrates the areas where myosin $\mathrm{VI}$ and MCP-1 immunofluorescence overlaps (yellow). These results demonstrate that hair cells express MCP-1. Magnification, $\times 630$.

contain detectable levels of MCP-1, RANTES, TNF alpha, sTNFR1, or any of the other cytokines present on the array (Fig. 3B, bottom; note that IL-4 and IL-5 were not tested in this experiment due to the sectioning of the array blot). Furthermore, control media with $4 \%$ serum (Fig. 3B, top) or without $4 \%$ serum (not shown) were also negative for the cytokines tested.

MCP-1 is expressed in inner ear cells. To explore the potential biological role of identified chemokines in the developing inner ear, immunocytochemistry was performed. MCP-1, RANTES, and TNF alpha were examined in paraffin-embedded tissues of embryonic and adult mouse inner ear tissues. The MCP-1, RANTES, and TNF alpha antibodies used in this study were not indicated for use in paraffin-embedded tissues by the manufacturer and attempts at examining these proteins in paraffin-embedded inner ear tissues resulted in inconclusive immunoreactivity in both the embryonic and adult mouse inner ear tissues. Therefore, primary cultures of dissociated E14 mouse inner cell cells were used. The E14 mouse inner ear

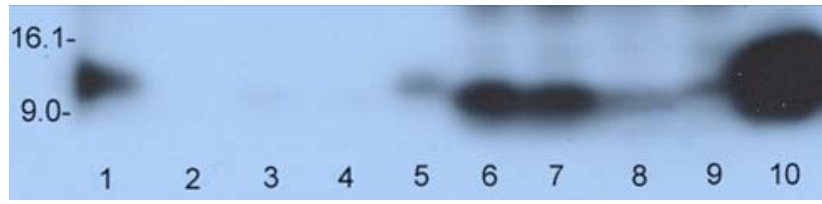

FIG. 5. Western blot analysis demonstrated MCP-1-like protein in active IMO2B1-conditioned medium when concentrated by filtration column chromatography. Lane 1: recombinant mouse MCP-1 (100 ng/ml; R\&D Systems); lane 2: IMO2B1-conditioned medium of (batch 4.22); lane 3: IMO2B1-conditioned medium (batch 4.25); lane 4: IMO2B1 (batch 4.28); lane 5: IMO2B1-conditioned medium (batch 5.11); lane 6: recombinant mouse MCP-1 (200 ng/ml, R\&D Systems); lane 7: IMO2B1-conditioned medium (batch 4.22 concentrated); lane 8: IMO2B1-conditioned medium (batch 4.25, concentrated); lane 9: IMO2B1-conditioned medium (batch 4.28 concentrated); lane 10: IMO2B1-conditioned medium (batch 5.11 concentrated). Molecular weight standards $(\mathrm{kDa})$ are indicated at the left. 


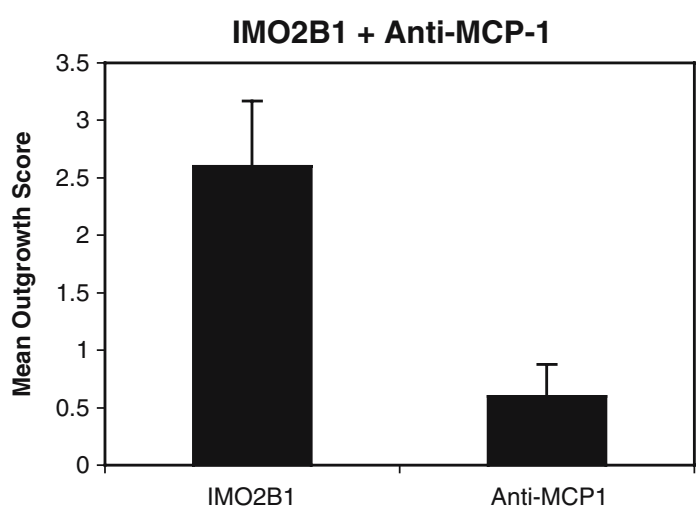

FIG. 6. E5 chick SAG treated with IMO2B1 produced neurite outgrowth (mean 2.6, SE 0.6, $n=5$, batch 9.22). Addition of $150 \mathrm{ng} /$ $\mathrm{ml}$ of anti-MCP-1 antibody to the IMO2B1 medium decreased outgrowth of SAG significantly (mean 0.6, SE 0.3, $n=5, p<0.01$, batch 9.22).

produces ODF bioactivity and our established assay enables easy identification of embryonic hair cells and supporting cells at this stage of development in cultured inner ear "domes" (Bianchi et al. 2002). RANTES and TNF alpha were below the level of detection in all samples examined, including samples that were double labeled with the hair cell marker myosin VI. In contrast, MCP-1 was highly expressed in aggregates of hair cells and supporting cells. Cultures that were double-labeled with a hair-cellspecific marker (anti-myosin VI antibody) and MCP-1 demonstrated that MCP-1 was prominently expressed in embryonic hair cells (Fig. 4). MCP-1 immunoreactivity was not detected in the cytokeratinexpressing supporting cells that surround the hair cells (Bianchi et al. 2002). MCP-1 reactivity was detected in some of the cells surrounding the hair cell-supporting cell aggregates (Fig. 4). Because these cells do not express myosin VI or cytokeratin (the supporting cell marker), they remain unidentified.

Western blot analysis (Fig. 5) confirmed that the antibody (R\&D Systems) detected MCP-1-like protein in both purified samples of mouse (Fig. 5) and human (not shown) MCP-1 (100-200 ng/ml, R\&D Systems, 279-MC) as well as in the bioactive IMO2B1 supernatants (batches 2.05, 4.22, 4.25, 4.28, 5.11, 9.13). IMO2B1-conditioned medium was concentrated using molecular weight cutoff filters. When molecular weight filters with $1-5 \mathrm{ml}$ of conditioned medium were used, only a faint band was detected at a molecular weight range that was slightly higher than that of the purified MCP-1. In contrast, when the $50 \mathrm{ml}$ of conditioned medium were concentrated over a $10-\mathrm{kDa}$ filter, prominent bands at the same size as purified MCP-1 were detected. In all samples, including negative controls lacking primary antibody, several higher molecular weight bands were also detected between approximately 50 and $100 \mathrm{kDa}$. These were likely due to nonspecific binding of the secondary antibody. However, because the IMO2B1conditioned medium is a complex mixture of proteins, the possibility that the primary antibody also detected higher molecular weight proteins or protein complexes cannot be ruled out.

Blocking MCP-1 activity reduces SAG outgrowth. To examine whether MCP-1 was a neurite-promoting component in the IMO2B1-conditioned medium, anti-MCP-1 function-blocking antibody (10-300 ng/ $\mathrm{ml}, \mathrm{R} \& \mathrm{D}$ Systems) was added to IMO2B1-conditioned medium. In the first series of experiments, outgrowth of E5 chick SAG explants was significantly inhibited when the IMO2B1-conditioned medium (batch 9.22) was treated with $150 \mathrm{ng} / \mathrm{ml}$ of anti-MCP-1 antibody (mean score 0.6, $n=5$, SE 0.3, $p<0.01$; Figs. 6 and 7). The limited amount of growth observed was similar to that observed in controls grown in unconditioned, defined culture medium (mean score 0.5, $n=9$ ). Addition of $75 \mathrm{ng} / \mathrm{ml}$ of anti-MCP-1 antibody produced similar reductions in growth (mean 0.5, $n=2$ ). Concentrations of this function-blocking antibody at 19 or $38 \mathrm{ng} / \mathrm{ml}$ decreased, but did not entirely eliminate, outgrowth (mean outgrowth 1.8 and 1.2, respectively, $n=2$ each). At higher concentrations $(300 \mathrm{ng} / \mathrm{ml})$, the MCP-1 antibody caused the SAG explants to lift from the surface of the dish and appear flat and thin $(n=2)$.

The effective concentration of anti-MCP-1 antibody varied with each preparation of IMO2B1conditioned medium, likely reflecting differences in the total amount of MCP-1 activity in each batch. For example, in a second series of experiments, $75 \mathrm{ng} / \mathrm{ml}$ of the anti-MCP-1 antibody led to SAG explants lifting from the surface of the culture well (IMO2B1 batch $4.29 ; n=3$ ). Controls treated with IMO2B1 (batch 1.90) and IgG at concentrations ranging from 1 to $200 \mu \mathrm{g} / \mathrm{ml}$ did not inhibit outgrowth of SAG (mean score $3.4 ; n=5$ ).

To test for nonspecific general neurotoxic effects of the antibody at the concentrations that reduced
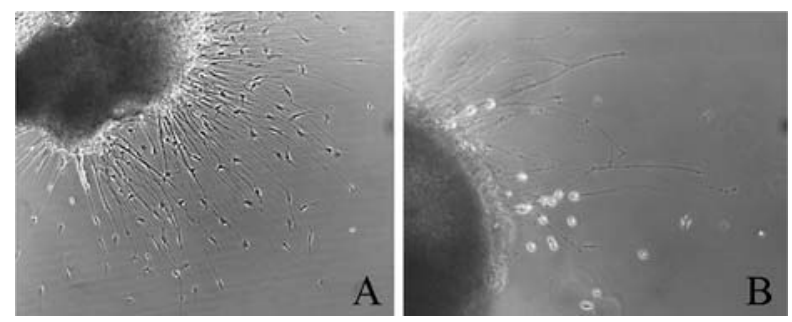

FIG. 7. (A) IMO2B1-conditioned medium promoted neurite outgrowth of E5 chick SAG (batch 9.22; explant score $=4$ ). (B) Addition of anti-MCP-1 antibody (150 ng/ml) decreased E5 chick SAG outgrowth (explant score $=1.5$ ). The photo shows the area of maximal outgrowth. Magnification, $\times 100($ A),$\times 200(B)$. 
SAG outgrowth, E8 DRG explants were grown in the presence of BDNF $(10 \mathrm{ng} / \mathrm{ml})$, a concentration that promotes DRG outgrowth, and anti-MCP-1 antibody was added. DRG explants continued to grow in the presence of BDNF with $75 \mathrm{ng} / \mathrm{ml}$ of anti-MCP-1 antibody (mean score 3.3, $n=3$, SE 0.8 ) or BDNF with $150 \mathrm{ng} / \mathrm{ml}$ of anti-MCP-1 antibody (mean 3.5, $n=4$, SE 3 ).

MCP-1 alone is insufficient to promote SAG outgrowth. To test the role of MCP-1 in SAG outgrowth further, MCP-1 (human or mouse recombinant, R\&D Systems) was added to cultures of E5 chick or E13 mouse SAG grown in control medium. However, no growth was noted when MCP-1 was added at concentrations ranging from 10 to $1000 \mathrm{ng} / \mathrm{ml}$ in any of these assays (E5 chick, $n=14$, human recombinant MCP-1; E13 mouse recombinant MCP-1, $n=9$, human MCP-1; E5 chick, $n=25$, mouse recombinant MCP-1; E14 mouse, $n=4$, mouse recombinant MCP1). This suggested that the MCP-1 was inactive or that MCP-1 may require additional factors to promote SAG outgrowth.

MCP-1 and IMO2B1-conditioned medium demonstrate chemotactic properties in a monocyte chemotaxis assay. To confirm that the MCP-1 used in the SAG assays retained bioactivity and that the function-blocking antibody both recognized MCP-1 and inhibited MCP-1 chemotactic activity, a standard, classic monocyte chemotactic assay was used. This assay also allowed testing of the chemotactic properties of

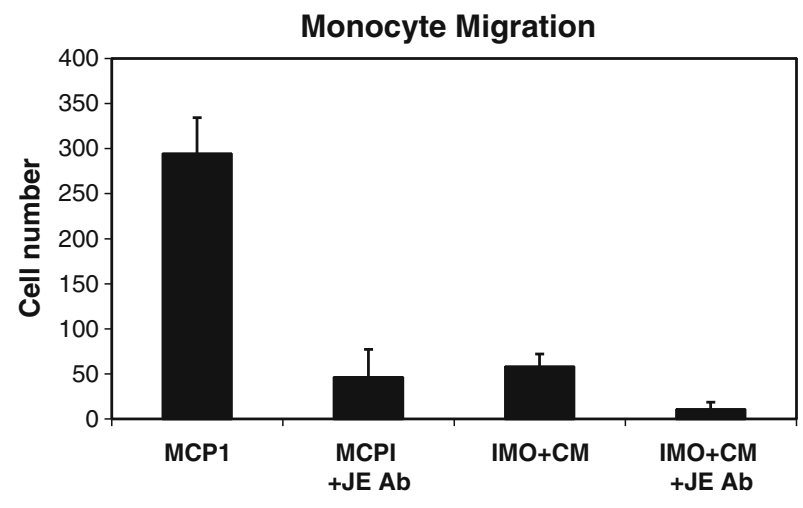

FIG. 8. Monocyte migration was measured using a classic chemotactic assay. The number of migrating cells is depicted minus the background number of cells migrating in control conditions (mean 58 cells, SE 19, $n=7$ ). In the presence of MCP$1(20 \mathrm{ng} / \mathrm{ml})$, monocytes migrated to the side of the chamber containing the protein (mean 299, SE, 40, $n=4$ ). Addition of the function-blocking anti-MCP-1 antibody reduced the migration of the monocytes significantly (mean 46, SE $31, n=3, p<0.01$ ). IMO2B1 alone also induced the migration of monocytes above background levels (mean 58, SE 14, $n=7$, batch 2.05) and the function-blocking antibody decreased this migration significantly (mean 11 , SE $8, n=$ $6, p<0.01$ ).
IMO2B1-conditioned medium. In this assay, the number of monocytes that migrate through a filter is determined in both experimental and control wells. The number of cells in the control wells is designated as "background" and the average number of these cells is subtracted from the total number of cells that migrate in the experimental wells (background mean in these experiments was 58 cells, $n=7$ ). In the presence of MCP-1 protein, monocytes migrated through a chemotactic chamber filter ( $8-\mu \mathrm{m}$ pore) as expected (Fig. 8; mean 299, SE $40, n=4)$, confirming that the MCP-1 was active. The anti-MCP-1 antibody reduced the migration of the monocytes significantly (Fig. 8; mean 46, SE $31, n=3$, $p<0.05$, Fig. 7) demonstrating that it too was bioactive and able to block MCP-1 chemotactic activity. IMO2B1-conditioned medium (batch 2.05) was also tested in these assays and demonstrated the ability to induce migration of monocytes at a level greater than background (Fig. 8; mean 58, SE 14, $n=$ 7). The anti-MCP-1 antibody also significantly inhibited migration of the monocytes treated with IMO2B1 (batch 2.05), further demonstrating the presence of MCP-1-like activity in the IMO2B1conditioned medium (Fig. 8; mean 11, SE $8, n=6$, $p<0.05$, Fig. 7). The IMO2B1-conditioned medium likely contains much lower levels of MCP-1 than the $20 \mathrm{ng} / \mathrm{ml}$ of purified MCP-1 used in these assays, which would account for the more modest chemotactic effect in wells treated with IMO2B1. However, it is important to note that the IMO2B1 has a statistically significant chemotactic effect on monocytes and

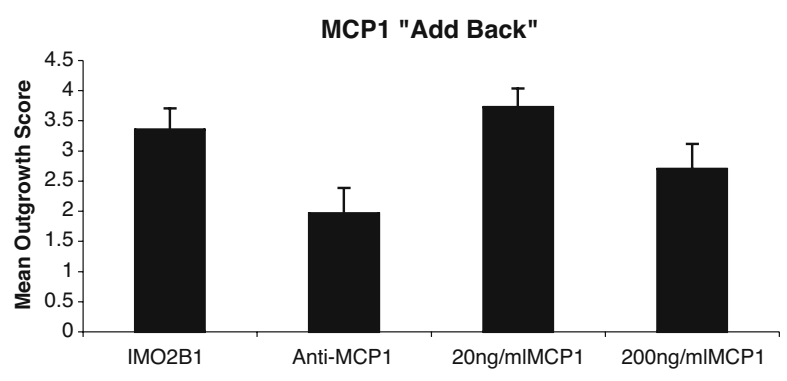

FIG. 9. IMO2B1-conditioned medium produced outgrowth from E5 chick SAG (IMO2B1 batch 9.13, mean 3.4, SE 0.35, $n=7$ ). When anti-MCP-1 function-blocking antibody was added at a concentration of 10 or $25 \mathrm{ng} / \mathrm{ml}(n=14$ total) the amount of outgrowth was reduced significantly (mean 1.97, SE 0.42, $n=14$, $p<0.01$ ). Supplementing the anti-MCP-1-treated IMO2B1-conditioned medium with MCP-1 ("add back", $20 \mathrm{ng} / \mathrm{ml}$ ) led to a statistically significant increase in SAG neurite outgrowth (mean $3.72, \mathrm{SE} 0.31, n=11, p<0.01$ ) above that observed with anti-MCP1 only, indicating a direct role for MCP-1 in promoting SAG outgrowth. No further increase was noted when $200 \mathrm{ng} / \mathrm{ml}$ of MCP-1 (mean 2.7, SE 0.42, $n=5 ; p>0.01$ ) was added to the antibody-treated cultures. 
that this migration can be blocked specifically by the anti-MCP-1 antibody.

MCP-1 as a cofactor for neurite outgrowth. The failure of MCP-1 alone to induce neurite outgrowth from SAG may have been because MCP-1 is only one of the neurite-outgrowth-promoting proteins in the IMO2B1-conditioned medium. To test for the interaction of MCP-1 with these putative, unidentified proteins, MCP-1 was added to cultures of E5 chick SAG treated with both IMO2B1-conditioned medium and anti-MCP-1 antibody. Anti-MCP-1 was added at a concentration of 10 or $25 \mathrm{ng} / \mathrm{ml}$. At these concentrations, anti-MCP-1 reduced, but did not eliminate all outgrowth, in the batches of IMO2B1-conditioned medium assayed (Fig. 9, batches 4.29 and 9.13, mean 1.97, SE 0.42, $n=14$ ). MCP-1 was then added to antibody-treated conditioned medium at a concentration of $20 \mathrm{ng} / \mathrm{ml}(n=11)$ or $200 \mathrm{ng} / \mathrm{ml} \quad(n=5)$. Under these conditions, $20 \mathrm{ng} / \mathrm{ml} \mathrm{MCP-1}$ was able to increase SAG outgrowth significantly above that observed in the presence of the IMO2B1-conditioned medium and anti-MCP-1 alone (batches 4.29 and 9.13, mean 3.7, SE 0.31, $n=11 ; p<0.05$; Fig. 9), whereas $200 \mathrm{ng} / \mathrm{ml}$ of MCP-1 did not increase growth in this assay (batch 9.13, mean 2.7, SE 0.42, $n=5, p>$ $0.05)$. The amount of neurite outgrowth from SAG treated with $20 \mathrm{ng} / \mathrm{ml}$ of MCP-1 was similar to that observed with IMO2B1-conditioned medium alone $(n=6$, Fig. 9). It is not clear why $200 \mathrm{ng} / \mathrm{ml}$ of MCP-1 did not promote growth further. It is possible that the decrease in growth reflects the typical doseresponse curve seen with MCP-1 and other growth factors at higher concentrations. Thus, MCP-1 (20 $\mathrm{ng} / \mathrm{ml}$ ) appears to be at least one of the active components of the IMO2B1-conditioned medium that is capable of interacting with other molecules to promote SAG outgrowth.

\section{DISCUSSION}

Sources of inner ear growth factors. Previous studies have demonstrated that the otocyst is a source of survival and neurite-promoting activity for early stage SAG (Ard and Morest 1984; Ard et al. 1985; Lefebvre et al. 1990; Bianchi and Cohan 1991, 1993a). However, the growth factor(s) required for initial outgrowth of SAG has not yet been fully characterized. Identifying the molecules that are active at early stages of SAG development is challenging due to the small size of the otocyst and the minute quantities of conditioned medium that are generated at any given time. For example, in order to produce ODF, eight E4-5 chick otocysts are cultured in $250 \mu \mathrm{l}$ of culture medium for 3 days. Due to the limited quantities of ODF generated by the otocyst, another source of ODF-like activity was needed.

Immortalized inner ear cell lines. The development of conditionally immortalized otocyst cells lines provided a source of unlimited amounts of conditioned medium and a potential source of ODF-like activity. In the present study, four IMO cell lines were found to promote SAG neurite outgrowth. A fifth cell line, IMO3A1, did not promote outgrowth of SAG.

Of the positive cell lines identified, the IMO2B1 cell line has been characterized most extensively (Thompson et al. 2003; Germiller et al. 2004). IMO2B1-conditioned medium promoted outgrowth of SAG explants and also promoted outgrowth and survival of dissociated SAG neurons similar to that observed with chick or mouse ODF. It is important to note that the cells of the IMO2B1 line maintain many of the characteristics of developing inner ear sensory epithelia (Gerlach et al. 2000; Thompson et al. 2003; Germiller et al. 2004; Barald and Kelley 2004). It is believed that the IMO2B1 cell line represents a multipotential precursor population that can give rise either to HC-like or SC-like cells under the influence of specific culture conditions (Germiller et al. 2004). The IMO2B1 cell line therefore appears to be an excellent source of material for identifying the neurite-promoting factor(s) released by cells of the early stage inner ear. As in all cases of cell-linederived materials, any molecular components identified in the cell lines have to be confirmed in the developing inner ear. So far, the cell lines have proven to be good predictors of results in the embryo (Thompson et al. 2003; Germiller et al. 2004).

Chemokines in inner ear cells. IMO2B1-conditioned medium was screened for cytokines/chemokines using a specific protein array. Cytokines/chemokines had not previously been investigated for SAG outgrowth-promoting activity. Chemokines consist of over 40 members that are classified into four distinct families based on the location of the cysteine residues (Mennicken et al. 1999). Each chemokine binds to one or more specific G-protein-coupled receptor. Chemokines have traditionally been associated with inflammatory responses, but recently have been associated with more diverse roles including regulating the migration and survival of neurons (Menniken et al. 1999; Chalasani et al. 2003a,b; Belmadani et al. 2005). For example, IL-8 promotes survival of hippocampal neurons (Araujo and Cotman 1993) and stromal-cell-derived factor 1 (SDF-1) promotes survival of retinal ganglion cells, even in the absence of neurotrophins (Chalasani et al. 2003b). SDF-1 also promotes the survival of sympathetic neurons and the receptor for SDF-1 is present in a number of neural populations (Chalasani et al. 2003b). RANTES has been found to influence the migration and differen- 
tiation of DRG neurons (Bolin et al. 1998) and MCP1 has been detected in the developing cerebellum where it has been suggested that it may regulate axonal outgrowth (Meng et al. 1999). Thus, several chemokine family members are implicated in regulating normal aspects of neural development.

Of the 22 cytokines/chemokines present in the commercial protein array examined in the present study, only 4 were detected in the IMO2B1-conditioned medium. The MCP-1 protein was consistently the most highly expressed in all batches of IMO2B1conditioned medium tested. MCP-1 is a member of the CC or beta family of chemokines and binds to the CCR2 and CCR4 chemokine receptors. In the present study, MCP-1 was also found to be prominently expressed in E14 mouse hair cells. Preliminary results suggest that one receptor, CCR2 is present in embryonic chick SAG neurons (unpublished observations); however, whether other chemokine receptors are present or differentially expressed has not yet been determined.

Chemokines as neurite-promoting factors. To test whether MCP-1 influenced SAG outgrowth, MCP-1 protein alone was added to cultures of chick or mouse SAG explants. However, no outgrowth was observed, at least at the concentrations evaluated. In contrast, addition of function-blocking antibody to the IMO2B1-conditioned medium decreased SAG outgrowth in a dose-dependent manner (10-150 $\mathrm{ng} / \mathrm{ml}$ ). The bioactivities of the MCP-1 and the antiMCP-1 antibody used in the assays were confirmed in a monocyte chemotaxis assay, indicating that the failure of MCP-1 to promote outgrowth was not due to inactive protein. The specificity of the antibody used in these studies was also confirmed by Western blot analysis. The antibody recognized purified mouse and human MCP-1 and detected an MCP-1like molecule in the bioactive IMO2B1-conditioned media. The intensity of the band detected in the IMO2B1-conditioned medium was greater when 50 ml IMO2B1-conditioned medium was concentrated over a $10-\mathrm{kDa}$ filter compared to $1-5 \mathrm{ml}$ being concentrated over 5- or $10-\mathrm{kDa}$ filters. This is not surprising as growth factors are typically produced in the picogram range making it difficult to detect such proteins, even following concentration of the supernatants. The protein array that first identified MCP-1 in the IMO2B1-conditioned medium was able to detect MCP-1 as low as $5 \mathrm{pg} / \mathrm{ml}$, whereas the antibody used in the Western blotting experiments could only detect MCP- 1 as low as $5 \mathrm{ng} / \mathrm{ml}$ under the reducing conditions. Because the IMO2B1-conditioned medium is a complex mixture of proteins and protein complexes, the possibility that additional proteins are detected by the function-blocking antibody cannot be entirely ruled out. However, consid- ering the above data as well as the observation that the antibody specifically blocked chemotactic activity of both purified MCP-1 and IMO2B1 in the monocyte assay, it seems unlikely that the anti-MCP-1-antibody blocked another protein in the IMO2B1-conditioned medium. It is also important to note that addition of other function-blocking antibodies to IMO2B1-conditioned medium including anti-TNF alpha and antiBDNF did not reduce the amount of outgrowth (unpublished observations).

Further support for the hypothesis that MCP-1 is a cofactor that promotes SAG outgrowth was obtained in experiments that demonstrated the addition of MCP-1 (20 ng/ml) to IMO2B1-conditioned medium treated with the function-blocking anti-MCP-1 antibody resulted in a medium that allowed SAG neurites to extend to lengths similar to SAG treated with only IMO2B1-conditioned medium. Thus, addition of MCP-1 "rescued" the antibody-treated SAG, allowing for neurite outgrowth to occur. It therefore appears that MCP-1 can produce outgrowth when SAG are provided with additional, as yet unidentified components found in the IMO2B1-conditioned medium. Addition of higher concentrations of MCP-1 (200 ng/ml) did not produce outgrowth greater than control levels. This may be a reflection of the sample size or a result of reaching a saturating level of MCP-1. In chemotactic assays, the most effective concentrations of the MCP-1 used in these studies ranged between 4-20 ng/ml (R\&D Systems).

Other chemokines such as SDF-1 have failed to directly attract retinal or DRG axons but appear to influence the activity of other molecules, including inhibitory proteins (Chalasani et al. 2003a). Further studies are under way to determine which other components(s) are present in the IMO-generated ODF, which are active at this stage of SAG outgrowth, and how they may interact with MCP-1. Neurotrophins are known to be required for survival and growth of later developmental-stage inner ear afferent neurons (Fritzsch et al. 1997; Tessarollo et al. 2004), and FGFs may potentiate some of the effects of neurotrophins (Hossain et al. 1997; Brumwell et al. 2000). It is not known whether MCP-1 interacts with neurotrophins or FGFs at earlier stages. Recent studies revealed that neurotrophins did not enhance the survival of retinal ganglion cells in the presence of the chemokine SDF1; nor did SDF-1 influence trk expression (Chalasani et al. 2003b). Thus, other molecules may interact with MCP-1 during the stage of initial SAG outgrowth. When IMO2B1-conditioned medium was run on polyacrylamide gels, a complex protein mixture was observed. Over 30 distinct bands were detected (Bianchi and Richards, unpublished observations; Molea and Barald, unpublished observations). Thus, 
there are a number of as yet unidentified potential proteins that may interact with MCP-1 to promote SAG outgrowth.

Several cytokines and chemokines have been observed in specific regions of the mature inner ear, particularly the lateral wall, where they are hypothesized to be involved in mediating an inflammatory response following infection or trauma to the inner ear (Yoshida et al. 1999; Ichimiya et al. 2000; Adams 2002). These results suggest multiple roles for MCP-1 in the inner ear, depending on its cellular localization and time of expression.

In summary, the present study reports the initial characterization of the first abundant source of ODFlike activity and the first observation of the chemokine MCP-1 as an inner-ear-derived, neurite-promoting molecule. Overall, the results of these experiments demonstrate the utility of the IMO cell lines in identifying candidate proteins that regulate inner ear development. The abundant quantities of conditioned medium that can be generated from the IMO cell lines will allow for both purification efforts to identify other biologically active proteins involved in outgrowth and survival of early-stage SAG, as well as the identification of specific proteins involved in other aspects of inner ear development.

\section{ACKNOWLEDGMENTS}

The authors thank Poornapriya Ramammurthy, Katie Au, Gabriel Hendricks, Malini Kochhar, Christopher Macklin, and Abigail Wallace for excellent technical assistance; Dr. Albert Borroni for assistance in preparing figures; Dr. Tama Hasson (UCSD) for providing the myosin VI antibody; and Drs. Chris Cohan, David Molea, and Matthew Kelley for helpful comments on the manuscript. This work was supported by NIH/NIDCD R15DC05587 and NSF0114831 (to LMB), NIH/NIDCD R01DC04184, and R01DC05939 (to KFB), and an Oberlin-University of Michigan Collaboration Award to $\mathrm{LMB}$ and $\mathrm{KFB}$.

\section{REFERENCES}

ADAms JC. Clinical implications of inflammatory cytokines in the cochlea: a technical note. Otol. Neurotol. 23:316-322, 2002.

Araujo DM, Cotman CW. Trophic effects of interleukin-4, -7 and -8 on hippocampal neuronal cultures: potential involvement of glial-derived factors. Brain Res. 600:49-55, 1993.

ARd MD, Morest DK. Cell death during development of the cochlear and vestibular ganglia of the chick. Neuroscience 2:535-547, 1984.

ARd MD, Morest DK, Hauger SH. Trophic interactions between the cochleovestibular ganglion of the chick embryo and its synaptic targets in culture. Neuroscience 16:151-170, 1985.

BARALD KF, Kelley MW. From placode to polarization: new tunes in development of the inner ear. Development 131:4119-4130, 2004.
Barald KF, Lindberg KH, Hardman K, Kava Ai, Lewis Je, Victor JC, Gardner CA, Poniatowski A. Immortalized cell lines from embryonic avian and murine otocysts: tools for molecular studies of the developing inner ear. Int. J. Dev. Neurosci. 15:523-540, 1997.

Belmandi AB, Tran PB, Ren D, Assimacopoulos S, Grove EA, Miller RJ. The chemokine stromal cell-derived factor-1 regulates the migration of sensory neuron progenitors. J. Neurosci. 25:3995-4003, 2005.

BiAnchi LM, Barald KF. Immortomouse otocyst cell lines as a source of neurite-promoting activity for statoacoustic neurons. Assoc. Res. Otolaryngol. Abstr. 645, 1998.

Bianchi LM, Cohan CS. Developmental regulation of a neuritepromoting factor influencing statoacoustic neurons. Dev. Brain Res. 64:167-174, 1991.

Bianchi LM, CoHan CS. Effects of the neurotrophins and CNTF on developing statoacoustic neurons: comparison with an otocystderived factor. 159:353-365, 1993a.

Bianchi LM, Cohan CS. Comparison of the biological activity of otocystderived factor to neurotrophins and FGF. Soc. Neurosci. Abstr. 19:922, 1993b.

Bianchi LM, Dolnick R, Medd A, Cohan CS. Developmental changes in growth factors released by the embryonic inner ear. Exp. Neurol. 150:98-108, 1998.

Bianchi LM, Person AL, Penney EB. Embryonic inner ear cells reaggregate into specific patterns in vitro. J. Assoc. Res. Otolatyngol. 3:418-429, 2002.

Bolin LM, Murray R, Lukacs NW, Strieter RM, Kunkel SL, Schall TJ, BACON KB. Primary sensory neurons migrate in response to the chemokine RANTES. J. Neuroimmunol. 81:49-57, 1998.

Brumwell CL, Hossain WA, Morest DK, Bernd P. Role for basic fibroblast growth factor (FGF-2) in tyrosine kinase (TrkB) expression in the early development and innervation of the auditory receptor: in vitro and in situ studies. Exp. Neurol. 162:121-145, 2000.

Chalasani SH, Sabelko KA, Sunshine MJ, Littman DR, Raper JA. A Chemokine, SDF-1, reduces the effectiveness of multiple axonal repellants and is required for normal axon pathfinding. J. Neurosci. 23:1360-1371, 2003a.

Chalasani SH, Baribaud F, Coughlan CM, Sunshine MJ, Lee VMY, Doms RW, LitTMan DR, Raper JA. The chemokine stromal cellderived factor-1 promotes the survival of embryonic retinal ganglion cells. J. Neurosci. 23:4601-4612, 2003b.

Coughlan CM, McManus CM, Sharron M, Gao Z-Y, Murphy D, Jaffer S, Choe W, Chen W, Hesselgesser J, Gaylord H, Kalyuzhny A, Lee M-Y, Wolf B, Doms RW, Kolson DL. Expression of multiple functional chemokine receptors and monocyte chemotactic protein-1 in human neurons. Neuroscience. 97:591$600,2000$.

Fritzsch B, Silo-Santiago I, Bianchi LM, Farinas I. Role of neurotrophic factors in regulating inner ear development. Trends Neurosci. 20:159-164, 1997.

Gerlach LM, Hutson Mr, Germiller JA, Nguyen-Luu D, Barald KF. Addition of the BMP4 antagonist, noggin disrupts avian inner ear development. Development 127:45-54, 2000.

Germiller JA, Smiley EC, Ellis AD, Hoff JS, Deshmukh I, Allen SJ, BARALD KF. Molecular characterization of conditionally-immortalized cell lines derived from early mouse embryonic inner ear. Dev. Dyn. 213:815-837, 2004.

Hossain WA, Morest DK. Fibroblast growth factors (FGF1, FGF-2) promote migration and neurite outgrowth of mouse co-chleovestibular ganglion cells in vitro: immunohistochemistry and antibody perturbation. J. Neurosci. Res. 62:40-55, 2000.

Hossain WA, Rutledge A, Morest DK. Critical periods of basic fibroblast growth factor and brain-derived neurotrophic factor in the development of the chicken cochleovestibular ganglion in vitro. Exp. Neurol. 147:437-451, 1997. 
Ichimiya I, Yoshida K, Hirano T, Suzuki M, Mogi G. Significance of spiral ligament fibrocytes with cochlear inflammation. Int. J. Ped. Otorhinolaryngol. 56:45-51, 2000.

Lefebvre PP, LePrince P, Weber T, Rigo J-M, Delree P, Moonen G. Neuronotrophic effects of the developing otic vesicle on cochleovestibular neurons: evidence for nerve growth factor involvement. Brain Res. 507:254-260, 1990.

Meng SZ, Ока A, Takashima S. Developmental expression of monocyte chemoattractant protein-1 in the human cerebellum and brainstem. Brain Dev. 21:30-35, 1999.

Menniken F, Maki R, de Souza E, Quirion R. Chemokines and chemokine receptors in the CNS: a possible role in neuroinflammation and patterning. TIPS 20:73-78, 1999.

Tessarollo L, Coppola V, Fritzsch B. NT-3 replacement with brainderived neurotrophic factor redirects vestibular nerve fibers to the cochlea. J. Neurosci. 24:2575-2584, 2004.

Thompson D, Gerlach-Bank LM, Barald KF, Koenig RJ. Retinoic acid repression of BMP4 in inner ear development. Mol. Cell. Biol. 23:2277-2286, 2003.

Yoshida K, Ichimiya I, Suzuki M, Mogi G. Effect of proinflammatory cytokines on cultured spiral ligament fibrocytes. Hear. Res. 137:155-159, 1999. 